\title{
Determination of absolute cross sections for cluster-specific decays
}

\author{
Andreas Hans ${ }^{1}$, André Knie ${ }^{1}$, Marko Förstel ${ }^{2,3}$, Philipp \\ Schmidt $^{1}$, Philipp Reiß ${ }^{1}$, Christian Ozga ${ }^{1}$, Uwe \\ Hergenhahn $^{2,4}$, and Arno Ehresmann ${ }^{1}$ \\ ${ }^{1}$ Institut für Physik, Universität Kassel, Heinrich-Plett-Str. 40, 34132 Kassel, \\ Germany \\ ${ }^{2}$ Max-Planck-Institut für Plasmaphysik, Wendelsteinstr. 1, 17491 Greifswald, \\ Germany \\ ${ }^{3}$ Department of Chemistry, University of Hawaii at Manoa, Honululu, HI 96822, \\ USA \\ ${ }^{4}$ Leibniz-Institut für Oberflächenmodifizierung e.V., Permoserstr. 15, 04318 \\ Leipzig, Germany \\ E-mail: hans@physik.uni-kassel.de,ehresmann@physik.uni-kassel.de
}

22 April 2016

\begin{abstract}
Fluorescence spectrometry is used to determine absolute cross sections of cluster-specific decay processes following photon excitation. Absolute values for selected processes in partially condensed supersonic jets are determined using atomic benchmarks. This method is applicable to a wide variety of processes in clusters in which a photon is emitted. In a proof-of-principle experiment, absolute cross sections of processes in two prototype systems were measured: I) inner-valence excitation and ionization of Ar clusters and II) resonant Interatomic Coulombic Decay (rICD) in Ne clusters. Example II is the first measurement of absolute cross sections of ICD whatsoever.
\end{abstract}

PACS numbers: $32.50 .+\mathrm{d}, 36.40 . \mathrm{Sx}$

Keywords: Cluster, Cross Sections, ICD, Fluorescence

Submitted to: J. Phys. B: At. Mol. Phys. 


\section{Introduction}

Experimentally determined absolute probabilities of photon-induced processes are indispensable for quantitative validations of theoretical models for the inter- ${ }_{60}$ 5 action of radiation with matter. A variety of methods and techniques for the determination of quantitative cross sections for fundamental processes, such as photoionization, photodissociation, and photoexcitation of isolated atoms, ions and molecules are well established $\mathrm{d}_{65}$ ${ }_{10}[1,2,3,4]$. This is not the case for agglomerates or clusters of these particles. It is a fundamental question how these values scale with the number of particles in a cluster and how properties evolve from isolated atoms to bulk matter [5]. For purely cluster-specific decay 15 paths, absent in the isolated species, absolute cross sections are necessary to include them in theoretical ${ }_{70}$ models. Some previous works concentrated on ionic clusters, and few data are available on cross sections (e.g. [6, 7]). Furthermore, experiments on positively ${ }_{20}$ and negatively charged fullerenes and endofullerenes have been carried out (e.g. [8,9]).

The quantification of absolute cross sections is of great value in many fields of research. For example, the interaction of clusters with cosmic radiation is

25 a fundamental issue in the physics of planetary atmospheres $[10,11]$. The chemistry of an atmosphere ${ }_{80}$ cloud formation, and the radiation budget are strongly affected by the composition of the atmospheric layers, the occurrence of clusters, and the probabilities of interaction with solar and cosmic photon radiation [12, 13]. Even a possible influence on the Earth's climate ${ }_{85}$ and an anti-greenhouse effect caused by clustered greenhouse gases have been discussed [14].

The interaction of clustered matter with ul35 traviolet and soft X-ray radiation became another area of interest when a novel non-local autoionization mechanism in weakly bound systems, Interatomic/Intermolecular Coulombic Decay (ICD), was predicted in 1997 [15] and observed experimentally 40 seven years later $[16,17]$. ICD was found to be a fundamental and omnipresent process in many systems. ICD and related processes, which are excitation and de-excitation pathways in clusters, have not yet been included in astrophysical models. One necessary con45 dition for including these processes in such models is the quantitative experimental determination of their $i_{00}$ probability. Currently there is no such work known to us.

Here, we describe a method for the determination 50 of absolute cross sections for photon-induced processes in clusters. We use fluorescence spectrometry after ${ }_{05}$ excitation by dispersed synchrotron radiation (photoninduced fluorescence spectrometry, PIFS [18]). This method is not constrained to the examples presented 55 in this work and independent from the process leading to photon emission. In general, the sensitivity to a certain process is given by the excitation mechanism and the sensitivity range of the used photon detector.

Earlier, we have shown that cluster-specific fluorescence emission can be separated from atomic lines [19]. The latter, with cross sections that often are well-known, are used as a calibration standard for the yet unknown cluster features. We demonstrate the method by applying it to the inner-valence excitation range of $\mathrm{Ar}$ and Ne clusters and determine the first absolute cross sections for a cluster-specific decay process, namely resonant Interatomic Coulombic Decay (rICD) in Ne clusters.

\section{Methods}

Experiments were carried out with partially condensed $\mathrm{Ne}$ and Ar cluster jets produced by supersonic expansion through a conical nozzle of $80 \mu \mathrm{m}$ diameter, $1.1 \mathrm{~mm}$ length, and $15^{\circ}$ half opening angle. The nozzle was cooled with liquid nitrogen for experiments with Ar and with liquid helium for those with Ne. By adjustment of the nozzle temperature and the pressure in the gas reservoir behind the nozzle, the mean cluster size $\langle N\rangle$ of the condensed beam is controlled. $\langle N\rangle$ is calculated using the expansion parameters and a scaling law introduced in [20]. All data shown below are valid for a jet generated with the given expansion parameters, resulting in a size distribution with mean cluster size $\langle N\rangle$. In the main chamber, the jet crossed the linearly horizontally polarized and monochromatized synchrotron radiation of the UE112PGM1 beamline of the Helmholtz-Zentrum Berlin, operating in single bunch mode ( 800 ns between two bunches). Exit slit widths of $300 \mu \mathrm{m}$ for $\mathrm{Ne}$ and $800 \mu \mathrm{m}$ for Ar, resulted in bandwidths of $30 \mathrm{meV}$ for photons in the $n$ s-subvalence threshold energy region of the respective element. A skimmer of $d=1 \mathrm{~mm}$ diameter separated the expansion chamber from the main chamber, and the crossing point of the jet and the synchrotron radiation was $z=5 \mathrm{~cm}$ behind the skimmer orifice. The main chamber was evacuated by a turbomolecular pump and had a background pressure without gas jet in the order of $5 \cdot 10^{-7} \mathrm{hPa}$. Fluorescence photons were detected undispersed in the plane spanned by synchrotron radiation and cluster jet under an angle of $45^{\circ}$ towards both beams using an open-face microchannel plate (MCP) detector. The experimental geometry is sketched in Fig. 1.

This detector is capable of detecting single photons with $\lambda_{\mathrm{fl}}<130 \mathrm{~nm}$ [19]. For cluster beam characterization, photoelectron spectra were recorded with a magnetic bottle electron spectrometer [21]. The exciting monochromatized synchrotron radiation was scanned stepwise over the energy region in which both 


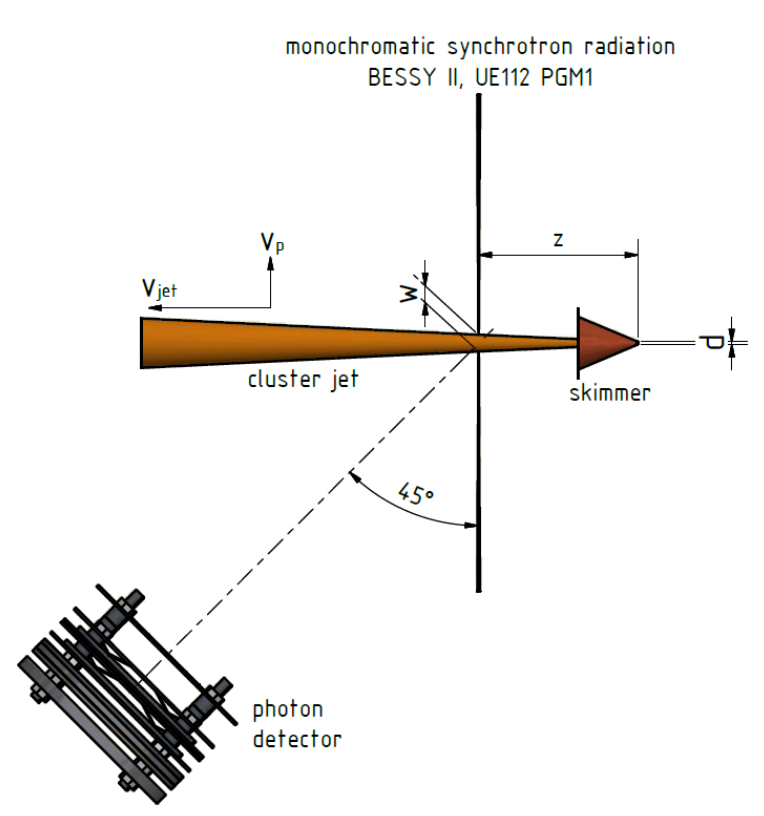

Figure 1. (color online) Sketch of the experiment geometry. The cluster jet enters the main chamber through a skimmer of diameter $d$. In the distance $z$ it interacts with a perpendicular beam of monochromatic synchrotron radiation. At this point, the jet has the width $w$, estimated from the velocities $v_{j e t}$ and $v_{p}$. The magnetic bottle electron spectrometer on top is not shown here.

cluster and atomic fluorescence emission are excited. This way, cluster fluorescence emission and atomic fluorescence emission can be distinguished despite the undispersed fluorescence detection.

The degree of condensation $K(\epsilon(0,1])$ is defined as the ratio of atoms condensed into clusters compared to the total amount of atoms in the jet. The total amount of atoms is the sum of the number of atoms condensed into clusters and the remaining free monomers, which are usually present since complete condensation of a cluster jet can hardly be achieved in practice. $K$ changes with different expansion parameters and moreover, for a single set of parameters, might differ if different parts of the cluster beam are probed. This can lead to an apparent change of $K$ if spectrometers with different solid angle of acceptance are compared to65 each other. In the present experiments, $K$ is measured by comparing monomer and cluster contribution to the electron spectra recorded with the magnetic bottle spectrometer. Both contributions can be separated by their different binding energies (see below).

Given $K(\epsilon(0,1])$, for every 100 condensed atoms $(100-100 \cdot K)$ individual uncondensed atoms are present and $(100 \cdot K) /\langle N\rangle$ clusters with a given mean 25 size $\langle N\rangle$. Therefore the emission intensity recorded from the clusters $\left(I_{\text {cluster }}\right)$ can be transferred tor5 an effective cross section per cluster $\left(\sigma_{\text {cluster }}\right)$ by multiplying the known emission cross section of the atoms $\sigma_{\text {atom }}$ by $(1-K) /(K /\langle N\rangle)$ and using the 30 observed intensity ratio $I_{\text {cluster }} / I_{\text {atom }}$ in:

$\sigma_{\text {cluster }}=\sigma_{\text {atom }} \cdot \frac{I_{\text {cluster }}}{I_{\text {atom }}} \cdot \frac{(1-K) \cdot\langle N\rangle}{K}$.

Depending on the explicit experimental case, further corrections to Eq. 1 might be necessary:

(I) We introduce a correction factor $E$ that takes into account different spectral detection efficiencies $\varepsilon(\lambda)$ of the fluorescence detector when fluorescence from clusters and atoms is emitted at different wavelengths:

$E=\frac{\varepsilon\left(\lambda_{\text {atom }}\right)}{\varepsilon\left(\lambda_{\text {cluster }}\right)}$.

40

(II) If the photon detector is not covering the same solid angle as the electron spectrometer, the degree of condensation $K$, determined from the electron spectra, must be corrected to an effective degree of condensation $\mathcal{K}$ seen by the fluorescence detector:

$\mathcal{K}=f(K)$

$f(K)$ is an experiment-specific transformation and can be regarded as 'solid angle correction'. As the degree of condensation $K$ is dependent on the solid angle of the detector (see above), $f(K)$ is given by the geometry of the experimental set-up and connects the degree of condensation measured by two detectors having a different solid angle with respect to the interaction volume of synchrotron radiation and sample.

Introducing these corrections Eq. 1 is modified to:

$\sigma_{\text {cluster }}=E \cdot \sigma_{\text {atom }} \cdot \frac{I_{\text {cluster }}}{I_{\text {atom }}} \cdot \frac{(1-\mathcal{K}) \cdot\langle N\rangle}{\mathcal{K}}$

If a partially condensed jet is not available, a reference gas may be added to a fully condensed cluster jet. In this case, the ratio $R$ between atoms of the reference gas and clusters of mean size has to be determined experimentally. Equations 3 and 4 are still valid, if $R$ is converted into a virtual degree of condensation

$K=1 /(R+1)$.

For both processes investigated, absolute cross sections for an atomic fluorescence emission process in the same exciting-photon energy range are available and the atomic and the cluster processes are excited by different energies, enabling the determination of absolute cluster fluorescence emission cross sections. For both of the individual noble gas atoms it is known that the subvalence $n$ s-electron hole, created by photoionization, is filled by an $n$ p-electron, undergoing fluorescence emission $n \mathrm{~s}^{1} n \mathrm{p}^{6}{ }^{2} \mathrm{~S}_{1 / 2} \rightarrow n \mathrm{~s}^{2} n \mathrm{p}^{5}{ }^{2} \mathrm{P}_{1 / 2,3 / 2}$ in the vacuum-ultraviolet (VUV) spectral range $(93.2 \mathrm{~nm}$ and $92.0 \mathrm{~nm}$ for $\operatorname{Ar}(n=3)$ and $46.23 \mathrm{~nm}$ and $46.07 \mathrm{~nm}$ for $\mathrm{Ne}(n=2))$. There is no 
competition of autoionization for either the Ne 2shole or the Ar 3s-hole fluorescence decays, because the respective energy releases are well below the atomic double ionization potentials. The $\mathrm{Ne} 2 \mathrm{~s}^{-}$ photoionization (threshold $48.48 \mathrm{eV}$ ) has been studied ${ }^{55}$ using photon-induced fluorescence spectrometry, and the fluorescence emission cross section as a function of the exciting-photon energy was quantified on an absolute scale $[22,23,24]$. The corresponding excitingthreshold in Ar atoms was subject to analogous investigations and absolute cross sections are also available $[25,26,27]$.

In the corresponding $\mathrm{Ne}$ and Ar clusters, atoms

15 are held together by the van-der-Waals force. The effect of the interatomic interactions on the electron orbitals is small and therefore the orbitals can still be assumed atomic-like. The ionization threshold in clusters, however, shifts to lower energies due to ${ }_{20}$ polarization screening in the final state. Corresponding shifts were observed for the thresholds of all higher lying shells [28]. This shift is experimentally well studied, and was found to depend on the cluster size $[29,30,31,32,33]$. For Ar clusters, a 25 pronounced structure of excitonic satellite states below the ionization threshold exists as well $[34,35,36$, 37]. However, the decay of inner-valence ionized Ar clusters occurs, analogously to the atomic case, solely by fluorescence emission because there is no excitation energies and because the absolute cross sections for atomic fluorescence emission are known, absolute cluster fluorescence emission cross sections can be determined.

35 In $\mathrm{Ne}$ clusters we apply the new method to fluorescence emission following resonant ICD [38]. As yet, there is no experimental estimate of absolute ${ }^{85}$ cross sections for non-resonant or resonant photon absorption with subsequent ICD decay whatsoever.

40 Here, we experimentally determine the cross section for a particular ICD process. This process has earlier been detected in Ne clusters by its subsequent characteristic ${ }^{90}$ fluorescence decay in the VUV spectral range [19]. The resonance structure after 2s-excitation of $\mathrm{Ne}$ clusters 45 has been investigated in more detail by means of ion spectroscopy [39]. After resonant photon absorption one atom in a cluster containing $N$ atoms is in $a^{95}$ excited state:

$\mathrm{Ne}_{N}+h \nu \longrightarrow \mathrm{Ne}^{*}\left(2 s^{1} 2 p^{6} n l\right) \mathrm{Ne}_{N-1}$.

${ }_{50}$ For this state there are two competing decay paths, local autoionization (AI) and resonant ICD (rICD) ${ }^{\text {jo }}$ [40]:

$$
\begin{aligned}
& \mathrm{Ne}^{*}\left(2 s^{1} 2 p^{6} n l\right) \mathrm{Ne}_{N-1} \\
& \longrightarrow \mathrm{Ne}^{+}\left(2 s^{2} 2 p^{5}\right) \mathrm{Ne}_{N-1}
\end{aligned}
$$

$(\mathrm{rICD}) \longrightarrow \mathrm{Ne}^{*}\left(2 s^{2} 2 p^{5} n l\right) \mathrm{Ne}^{+}\left(2 s^{2} 2 p^{5}\right) \mathrm{Ne}_{N-2}$.

The energy stored in the excited Ne atom after rICD is not sufficient for further (auto-)ionization, and a photon in the VUV spectral range is emitted [19]. For this example the ICD channel is the only one leading to fluorescence, because the AI channel leads to the ionic ground state. The measured fluorescence emission cross section equals therefore the differential cross section of photon absorption and subsequent ICD.

The acceptance volumes of our photon detector and electron spectrometer are not identical. The electron spectrometer accepts electrons only from a small volume around the crossing point of synchrotron and cluster beam. The acceptance volume of the photon detector extends by about $5 \mathrm{~cm}$ in both directions along the synchrotron radiation beam. Therefore, some of the fluorescence signal results from ionization of the background gas. As a result, the degree of condensation observed by the electron spectrometer differs from the one observed by the photon detector. This motivates the introduction of the correction function $f(K)$ described earlier and the degree of condensation is corrected to an effective value as described in Ref. [41]. In both cases ( $\mathrm{Ar}$ and Ne), the fluorescence emission features from atoms and clusters are in the VUV spectral range and do not differ much in their emission wavelength. The detection efficiency of the MCP is almost constant in this range of emission wavelength $[42,43]$ and its uncertainty well below other uncertainties influencing the results. Therefore, no correction is necessary $(E=1)$.

\section{Results}

Figure 2(a) shows the VUV photon emission of an atomic Ar jet as a function of the exciting-photon energy. The displayed data is background-corrected and the steep increase in fluorescence intensity at about $29.2 \mathrm{eV}$ corresponds to the 3s-electron photoionization threshold [26]. No other VUV fluorescence transitions within the sensitivity range of the detector are known for these exciting-photon energies. The right hand side ordinate was calibrated to $0.87 \mathrm{Mb}$ at $29.4 \mathrm{eV}$ according to Ref. [26], indicated by the black arrow in Fig. 2(a). Figure 2(b) shows the analogous data for a cluster jet produced at 330 mbar stagnation pressure and $113 \mathrm{~K}$ nozzle temperature. The intensity function shows features (labeled 1 in Fig. 2(b)) below $28.2 \mathrm{eV}$, an almost constant intensity from about $28.2 \mathrm{eV}$ (labeled 2) towards higher exciting-photon energies, and a steep increase at $29.2 \mathrm{eV}$. The latter is caused by the remaining free atoms in the only partially condensed jet. The other features of the fluorescence excitation function are absent in measurements of 


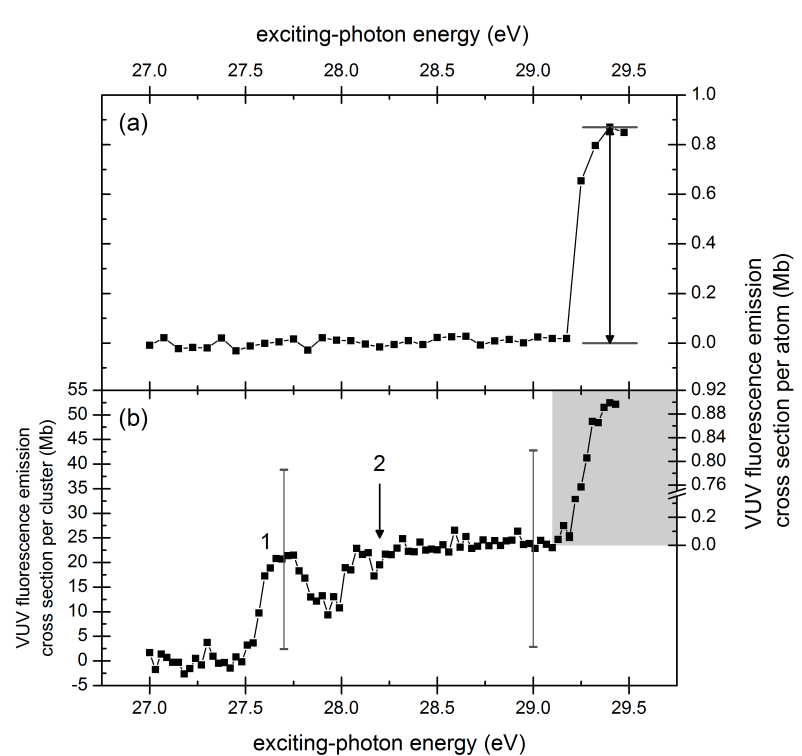

Figure 2. (a) Photon-induced VUV fluorescence $\left(\lambda_{\mathrm{fl}}<130 \mathrm{~nm}\right)$ from an atomic Ar jet vs. excitation energy. The absolute cross section scale was calibrated to the data of Ref. [26] at $29.4 \mathrm{eV}$. (b) Analogous data for a partially condensed jet with a mean cluster size of $\langle N\rangle=36$. The right hand side ordinate is valid ${ }^{50}$ for the shaded region and corresponds to the 3s-photoionization cross section of isolated atoms ((a), [26]). The left hand side ordinate (white region) indicates the VUV fluorescence emission cross sections for clusters with the given mean size. Features 1 and 2 are discussed in the text.

isolated atoms and are therefore attributed to emission by clusters. The increase in fluorescence intensity at $29.2 \mathrm{eV}$ corresponds, on an absolute scale, to the same cross section increase observed for the pure atomic 5 jet: $0.87 \mathrm{Mb} \pm 18 \%$ per atom at $29.4 \mathrm{eV}[26]$. The right hand side ordinate of Fig. 2(b) is calibrated using this value and is valid for the signal increase from uncondensed $\mathrm{Ar}$ in the shaded region. This increase can be seen on top of the almost constant cluster signal

10 background. A photoelectron spectrum of the cluster jet was recorded at a photon energy of $52 \mathrm{eV}$. The degree of condensation was determined as $0.7 \pm 0.1$. A mean cluster size of $\langle N\rangle=36$ was calculated using the procedure of Ref. [20]. After the experiment-specific corrections, the effective degree of condensation $\mathcal{K}$ seen by the photon detector is $0.4 \pm 0.1$. The absolute fluorescence emission cross sections were calculated using Eq. 4. These values are displayed on the left hand side ordinate of Fig. 2(b) and are valid for all 20 features absent in the pure atomic signal (not shaded region). The absolute emission cross section per $\mathrm{Ar}$ cluster below the atomic threshold is in the range of $(23 \pm 18) \mathrm{Mb}$ at $29.2 \mathrm{eV}$ for $\langle N\rangle=36$. Two representative error bars are shown at $27.7 \mathrm{eV}$ and $_{80}$ $2529.0 \mathrm{eV}$.

The onset of 3s-photoelectrons from the largest clusters (with lowest binding energy) in the electron spectrum is at approximately $28.2 \mathrm{eV}$. Above this energy, labeled 2 in Fig. 2(b), VUV photon emission ${ }_{3}$ after 3s-ionization in clusters can contribute to the cross section. Feature 1 at lower exciting-photon energies cannot be initiated by 3s-ionization as there is no corresponding photoelectron signal. Clusterspecific photoelectron satellites below the 3s-ionization threshold have been observed before which may lead to VUV fluorescence by decay of excitonic states $[34,35,36,37]$. Above the 3s-ionization threshold of clusters, still contributions from other channels are likely, because the pure signal intensity from $3 p \rightarrow 3 s$ after 3s-ionization at any energy $E$ is expected to be the integral of the photoelectron spectrum below $E$. An unambiguous distinction of the contribution of these induvidual processes to the observed features is not possible with this undispersed measurement. We therefore state that the calculated cross sections are values for VUV photon emission, possibly a result of several processes which overlap in the excitation energy range for clusters of different size in the jet. The emission cross section per atom in a cluster at $29.2 \mathrm{eV}$ is $(23 \pm 18) \mathrm{Mb} / 36$ atoms $=(0.64 \pm 0.50) \mathrm{Mb} /$ atom, which is in the range of the 3s-electron emission cross section for an individual atom within the uncertainty of our experiment. However, due to the relative large uncertainty a contribution from other channels can still not be excluded.

Figure 3 shows the results for Ne. In panel (a) the VUV photon emission of an atomic jet is shown. The 2s-ionization threshold is calibrated to a cross section of $0.17 \mathrm{Mb} \pm 25 \%$ per atom at $48.6 \mathrm{eV}$ [23]. The narrow, not well resolved peaks below the threshold correspond to excitation of atomic high $n$ $1 \mathrm{~s}^{2} 2 \mathrm{~s}^{1} 2 \mathrm{p}^{6} n \mathrm{p}$ resonances, whose fluorescence decays $2 \mathrm{~s}^{1} 2 \mathrm{p}^{6} n \mathrm{p} \rightarrow 2 \mathrm{~s}^{2} 2 \mathrm{p}^{5} n \mathrm{p}$ close to $46 \mathrm{~nm}$ can compete with autoionization [44]. The analogous result for a cluster jet is shown in Fig. 3(b), recorded for an mean cluster size of $\langle N\rangle=20$ [20] with a stagnation pressure of 520 mbar and a nozzle temperature of $60 \mathrm{~K}$. The fluorescence emission function shows two broader features (labeled 1 and 2 in Fig. 3(b)), which have recently been shown to stem from resonant ICD [19]. The ionization threshold of the remaining free atoms can be identified at about $48.5 \mathrm{eV}$. Below this exciting-photon energy, no intensity plateau from cluster fluorescence is visible, therefore the right hand side ordinate of Fig. 3(b) can be calibrated as done for panel (a). The photoelectron spectrum of the cluster jet was recorded at $61 \mathrm{eV}$. We determined a degree of condensation of $K=0.5 \pm 0.1$ and the effective degree of condensation seen by the photon detector is $\mathcal{K}=0.3 \pm 0.1$. Absolute cross sections for the cluster fluorescence features 1 and 2 of Fig. 3(b) can 


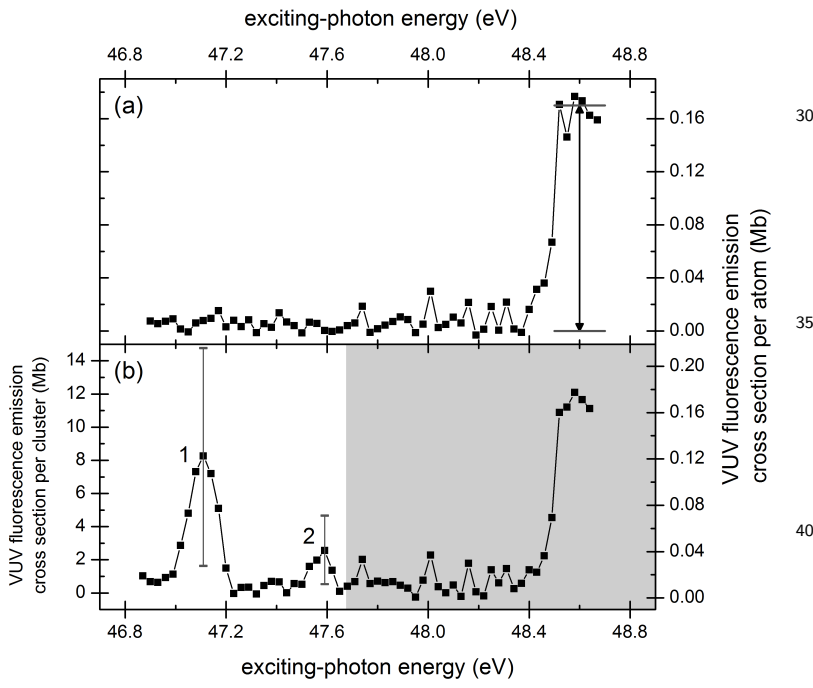

Figure 3. (a) VUV fluorescence $\left(\lambda_{\mathrm{fl}}<130 \mathrm{~nm}\right)$ of an atomic Ne jet as a function of the excitation energy. The absolute cross section scale was calibrated according to Ref. [23] at $48.6 \mathrm{eV} .45$ (b) Analogous measurement for a partially condensed jet with a mean cluster size of $\langle N\rangle=20$. The right hand side ordinate is valid for the shaded region and corresponds to the 2 s-electron photoionization cross section of isolated atoms ((a), [23]), the left hand side ordinate (white region) corresponds to VUV fluorescence emission cross sections of clusters of mean size of $_{50}$ 20 atoms. Features 1 and 2 are discussed in the text.

be deduced in analogy to the Ar case using Eq. 4, resulting in peak values of $(8.2 \pm 6.6) \mathrm{Mb}$ for feature 1 and $(2.6 \pm 2.1) \mathrm{Mb}$ for feature 2 . The scale at the left hand side ordinate is valid for cluster emission.

5 Representative error bars for features 1 and 2 are $^{55}$ shown. Here we measured the first absolute cross sections for an ICD process. The contribution of individual atoms to the peak intensities in Fig. 3(b) is $(8.2 \pm 6.6) \mathrm{Mb} / 20$ atoms $=(0.41 \pm 0.33) \mathrm{Mb} /$ atom $^{60}$ 10 for feature 1 and $(2.6 \pm 2.1) \mathrm{Mb} / 20$ atoms $=$ $(0.13 \pm 0.11) \mathrm{Mb} /$ atom for feature 2 .

The relative uncertainties of the given absolute values were estimated to be $80 \%$, including the uncertainties from the degree of condensation, determined with the electron spectrometer $(\approx 15-20 \%)$, and its correction due to experiment-specific parameters $(\approx 25-35 \%)$ and the uncertainties given for the atomic ${ }^{70}$ benchmarks $(\approx 20-25 \%,[26,23])$. However, due to a high signal to noise ratio, the statistical uncertainties are below $25 \%$. The presented values are the first quantitative fluorescence emission cross sections from clusters. The largest contribution to the uncertainty is caused by the necessary corrections for different solid angles of the electron spectrometer and photon detec-

or. In a future advanced experiment, this uncertainty could be reduced dramatically, if the set-up is specially designed for this purpose.

\section{Conclusion}

Summarizing, we present absolute cross sections for the excitation of fluorescing states in clusters by monochromatized synchrotron radiation. Among the different features observed by cluster fluorescence emission were excitonic photoelectron satellites, features corresponding to shifted ionization thresholds of clusters with respect to isolated atoms and, in the case of Ne clusters, two features corresponding to resonant ICD processes with subsequent fluorescence emission. Cross sections were determined by comparing signals stemming from clusters to known absolute subvalence $n$ s-shell ionization cross sections of isolated atoms, which are present in the mixed atom/cluster jets.

\section{Acknowledgments}

The work was supported by the Hesse State Initiative for the Development of Scientific and Economic Excellence (LOEWE) in the LOEWE-Focus Electron Dynamics of Chiral Systems (ELCH) and by a BMBF grant under promotion code number 05K13RK1. We thank HZB for beamtime allocation, the BESSY staff for support, and Felix Lelievre for assistance during the beamtime. Funding by the Deutsche Forschungsgemeinschaft via the Forschergruppe 1789 is gratefully acknowledged.

\section{References}

[1] V. Schmidt, Rep. Prog. Phys. 55, 1483 (1992).

[2] H. Kjeldsen, J. Phys. B 39, R325 (2006).

[3] S. Schippers et al., J. Phys. B 47, 115602 (2014).

[4] M. Glass-Maujean et al., Phys. Rev. Lett. 104, 183002 (2010).

[5] M. Förstel et al., Phys. Rev. B 82, 125450 (2010).

[6] H. Haberland et al., Phys. Rev. Lett. 67, 3290 (1991).

[7] P. Ayotte and M. A. Johnson, J. Chem. Phys. 106, 811 (1997).

[8] R. C. Bilodeau et al., Phys. Rev. Lett. 111, 043003 (2013).

[9] A. Müller et al., Phys. Rev. Lett. 101, 133001 (2008).

[10] A. W. Castleman, in Heterogeneous Atmospheric Chemistry, edited by D. R. Schryer (American Geophysical Union, Washington, D.C., 1982).

[11] H. S. W. Massey and D. R. Bates, Applied Atomic Collision Physics (Academic Press, New York, 1982), Vol. I.

[12] M. Kulmala, L. Pirjola, and J. M. Mäkelä, Nature (London) 404, 66 (2000)

[13] J. M. Anglada et al., J. Phys. Chem. A 117, 10381 (2013).

[14] A. Galashev and O. Rakhmanova, IOP Conf. Series: Earth Environ. Sci. 6, 282025 (209).

[15] L. S. Cederbaum, J. Zobeley, and F. Tarantelli, Phys. Rev. Lett. 79, 4778 (1997).

[16] S. Marburger, O. Kugeler, U. Hergenhahn, and T. Möller, Phys. Rev. Lett. 90, 203401 (2003).

[17] T. Jahnke et al., Phys. Rev. Lett. 93, 163401 (2004).

[18] H. Schmoranzer, H. Liebel, F. Vollweiler, R. MüllerAlbrecht, A. Ehresmann, K.-H. Schartner, and B. Zimmermann, Nucl. Instrum. Methods Phys. Res. A 467, 1526 (2001).

[19] A. Knie et al., New J. Phys. 16, 102002 (2014). 
[20] O. F. Hagena, Surf. Sci. 106, 101 (1981).

[21] M. Mucke et al., Nat. Phys. 6, 143 (2010).

[22] K.-H. Schartner, B. Magel, B. Möbus, H. Schmoranzer, and M. Wildberger, J. Phys. B 23, L527 (1990).

5 [23] K.-H. Schartner, B. Möbus, G. Mentzel, A. Ehresmann, F. Vollweiler, and H. Schmoranzer, Phys. Lett. A 169, 393 (1990).

[24] O. Wilhelmi et al., J. Electron Spectrosc. Relat. Phenom. 101, 155 (1999).

10 [25] K.-H. Schartner, B. Möbus, P. Lenz, H. Schmoranzer, and M. Wildberger, Phys. Rev. Lett. 61, 2744 (1988).

[26] B. Möbus et al., Phys. Rev. A 47, 3888 (1993).

[27] O. Wilhelmi et al., Phys. Lett. A 228, 283 (1997).

[28] O. Björneholm et al., J. Chem. Phys. 104, 1846 (1996).

15 [29] E. Rühl et al., Chem. Phys. Lett. 191, 430 (1992).

[30] W. Kamke et al., Z. Phys. D 14, 339 (1989).

[31] G. Ganteför et al., J. Chem. Phys. 91, 7972 (1989).

[32] Öhrwall et al., Phys. Rev. Lett. 93, 173401 (2004).

[33] S. Barth, M. Ončák, V. Ulrich, M. Mucke, T. Lischke, P. Slavíček, and U. Hergenhahn, J. Phys. Chem. A 113, 13519 (2009).

[34] J. de Vries et al., J. Chem. Phys. 101, 2372 (1994).

[35] R. Thissen et al., Eur. Phys. J. D 4, 335 (1998).

[36] J. Wörmer et al., J. Chem. Phys. 104, 8269 (1996).

25 [37] U. Hergenhahn et al., Chem. Phys. Lett. 351, 235 (2002).

[38] S. Barth, S. Joshi, S. Marburger, V. Ulrich, A. Lindblad, G. Öhrwall, O. Björneholm, and U. Hergenhahn, J. Chem. Phys. 122, 241102 (2005).

[39] R. Flesch, N. Kosugi, A. Knop-Gericke, and E. Rühl, Z. Phys. Chem. 228, 387 (2014).

[40] S. Kopelke, K. Gokhberg, L. S. Cederbaum, and V. Averbukh, J. Chem. Phys. 130, 144103 (2009).

[41] See Supplemental Material at [URL] for detailed description of the correction procedure.

35 [42] O. H. W. Siegmund, in Vacuum Ultraviolet Spectroscopy II, edited by J. A. R. Samson and D. L. Ederer (Academic Press, San Diego, 2000).

[43] Hamamatsu Photonics, in MCP Assembly technical information (Shimokanzo, Japan, 2006).

40

[44] P. Lablanquie et al., Phys. Rev. Lett. 84, 431 (2000).

[45] W. Christen, K. Rademann, and U. Even, J. Phys. Chem. A 114, 11189 (2010). 